Int. J. Dev. Biol. 59: 205-209 (2015)

doi: $10.1387 / \mathrm{ijdb} .140348 \mathrm{mj}$

\title{
Generation and characterization of mice harboring a conditional CXCL12 allele
}

\author{
RYAN E. HILLMER ${ }^{1}$, JOANNE P. BOISVERT², MONICA J. CUCCIARE ${ }^{1}$, MICHAEL B. DWINELL*,2 \\ and MILAN JOKSIMOVIC*,1 \\ ${ }^{1}$ Department of Cell Biology, Neurobiology and Anatomy, ${ }^{2}$ Department of Microbiology and Molecular Genetics, \\ Medical College of Wisconsin, Milwaukee, WI, USA
}

\begin{abstract}
The chemokine CXCL12 has important functions in immune and central nervous systems. Moreover, a global disruption of CXCL12 in mice results in perinatal lethality. To circumvent this impediment and provide a tool for analyzing CXCL12 functions in specific organ systems, we have generated a mouse line harboring a loxP-site flanked exon 2 of $C X C L 12$. A germ line deleter, $\beta$ actin::cre was used to remove a CXCL12 exon 2 and subsequently systemic CXCL12 exon 2 deficient embryos were generated. These mutant embryos showed a marked depletion of CXCL12 transcript. As expected from the global mutant phenotype, our mutants were also characterized by highly irregular cerebellar cytoarchitecture of the external granule layer as well as altered radial migration of midbrain dopaminergic neurons. Importantly, migration of the pontine grey nucleus (PGN) was derailed and remarkably resembled the global mutant phenotype of the CXCL12 receptor - CXCR4 in this system. Despite the fact that CXCL12 signaling can be mediated through receptors other than CXCR4, our results indicate a monogamous relationship between the CXCL12 ligand and CXCR4 receptor in controlling PGN migration. Our findings further expand on the understanding of CXCL12 function in PGN development. Moreover, phenotypic similarities between our mutants and mice harboring a global CXCL12 disruption support the validity of our line. Importantly, these results strongly suggest that our conditional $C X C L 12$ line can be used as a powerful tool to manipulate CXCL12 signaling and function in vivo.
\end{abstract}

KEY WORDS: SDF1 floxed, pontine grey nucleus, external granule layer, midbrain dopaminergic neuron

\section{Introduction}

Chemokines are a class of small molecules that were initially found to function as leukocyte attractants in the immune system (Baggiolini, 1998). Apart from this system, chemokines also impact hematopoiesis, lymphopoesis, angiogenesis and neuron migration in the central nervous system (CNS) (Bodea et al., 2014; Ma et al., 1998; Nagasawa et al., 1996; Sánchez-Alcañiz et al., 2011; Wang et al., 2011; Yang et al., 2013).

One of the chemokine ligands, stromal cell-derived factor 1 (SDF-1; CXCL12) binds receptors CXCR4 and CXCR7 (Balabanian et al., 2005; Oberlin et al., 1996). In the developing CNS, CXCL12 regulates neuron migration and proliferation (Sánchez-Alcañiz et al., 2011; Wang et al., 2011; Zou et al., 1998). For instance, the cerebellum of CXCR4 or CXCL12 mutant mice is characterized by ectopically located Purkinje cells and irregular external granule layer (EGL), which protrudes into the cerebellar parenchyma (Ma et al., 1998; Vilz et al., 2005). In the midbrain, CXCR4 is expressed in postmitotic midbrain dopaminergic (mDA) neurons, whereas CXCL12 is expressed in meninges. Consistently, a global disruption of CXCR4 or CXCL12 transiently alters radial migration of $\mathrm{mDA}$ neurons wherein CXCL12/CXCR4 signaling appears to modulate the migration efficiency of mDAneurons (Bodea et al., 2014; Yang et al., 2013). In the hindbrain, the pontine grey nucleus (PGN) neurons migrate anteriorly beneath the pial surface and settle down in the

\footnotetext{
Abbreviations used in this paper: CXCL12; chemokine(C-X-C motif) ligand 12; CXCR4, chemokine (C-X-C motif) receptor 4; CXCR7, atypical chemokine receptor 3 ; ECN, external cuneate nucleus; EGL, external granule layer; LRN, lateral reticular nucleus; mDA, midbrain dopaminergic; PGN, pontine grey nucleus.
} 
ventral anterior hindbrain, in contrast to posterior migration of the lateral reticular nucleus (LRN) and external cuneate nucleus (ECN) neurons (Rodriguez and Dymecki, 2000). These migrating neurons express CXCR4, whereas CXCL12 is expressed in surrounding meninges. Consistent with this expression pattern, CXCR4 mutants are characterized by derailed PGN neuron migration and reduced size of this nucleus in contrast to the intact posterior migration of the LRN and ECN (Vilz et al., 2005; Zhu et al., 2009).

Here, we generated a mouse line bearing a floxed exon 2 of the CXCL12 gene. Mutants with a conditional deletion of both alleles show aberrant development of the cerebellum, mDA neurons, and $P G N$ that is strikingly similar to phenotypes in corresponding structures of global CXCL12 or CXCR4 mutants.

\section{Results}

\section{Generation of mice bearing a conditional CXCL12 allele}

Analyses of global CXCL12 mutants generated by insertional mutagenesis are restricted to embryonic development due to perinatal lethality (Ma et al., 1998; Nagasawa et al., 1996; Zhu et al., 2009; Zhu et al., 2002). Accordingly, we sought to generate a conditional CXCL12 allele that can be used as a tool to target its specific developmental and postnatal functions and characterized this line based on established neural phenotypes of mice with disrupted CXCL12 signaling.

CXCL12 binds its cognate receptor CXCR4 through exon 2 sequences (Crump et al., 1997). Thus, removing this exon should prevent any corresponding downstream signaling. Accordingly, we positioned loxP sites to flank exon 2 (Figs. 1A, S1, and S2). Furthermore, we reasoned that homozygous, $C X C L 12^{\Delta e \times 2 / \Delta e \times 2}$ mutant embryos lacking both alleles of exon 2 should recapitulate a global CXCL12 mutant phenotype (Nagasawa et al., 1996). To generate these mutants, we first crossed floxed CXCL12 exon 2 animals $\left(C X C L 12^{F /+}\right)$ to a germ line deleter $\beta$ actin::cre. Resulting animals heterozygous for an exon 2 deletion $\left(C X C L 12^{+/ \Delta \text { ex2 }}\right)$ were then intercrossed to generate $C X C L 12^{\Delta e x 2 / \Delta e x 2}$ embryos (Fig. S3A). All animals and embryos were genotyped by PCR analysis (Fig. S3B). To address if a deletion of exon 2 of the CXCL12 gene results in a loss-of-function mutation, we performed RNA in situ hybridization using a $C X C L 12$ riboprobe (Fig. 1A; dark blue line).

A
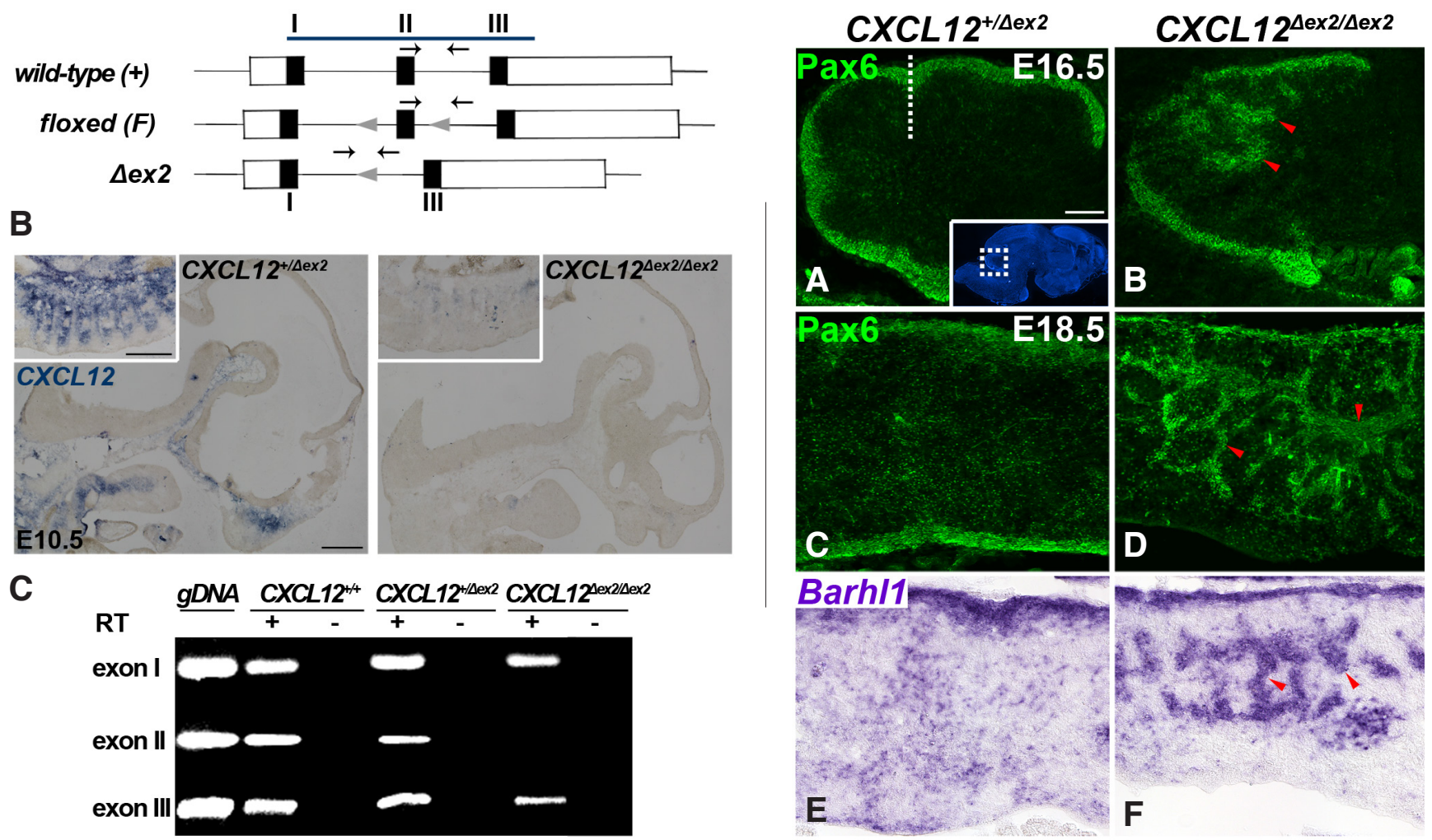

Fig. 1 (Left). Generation of the CXCL12 floxed line. (A) Schematic of the CXCL12 genomic locus. Exons (I-III) and introns are depicted by black boxes and solid lines, respectively. The 5'UTR and 3'UTR are indicated by open boxes. A position of the primers used for genotyping (Supp. Fig. S3B) is indicated by black arrows. Grey arrowheads indicate loxP sites. The dark blue line indicates the position of the CXCL12 riboprobe used in (B). (B) RNA in situ hybridization on sagittal sections for CXCL12. Somite regions of the corresponding genotypes are shown in insets. Anterior is to the right, posterior to the left. (C) RT-PCR analysis of control and mutant samples. gDNA, genomic DNA; RT, reverse transcriptase. Scale bars, 500 um.

Fig. 2 (Right). CXCL12 $2^{\operatorname{sex} / \Delta e \times 2}$ embryos exhibit aberrant cerebellar organization. Immunofluorescent labeling for Pax6 (A-D) and RNA in situ hybridization using a Barhl1 riboprobe (E,F) reveal a disorganized external granule layer (EGL) in mutant (B,D,F) (red arrowheads), in comparison to control embryos (A, C,E) in sagittal (A,B) and coronal sections (C-F). (A) The dashed white line indicates the relative axial level of coronal sections shown in $(C-F)$. Inset in (A) is a DAPl labeled sagittal section in which the white dotted box indicates the approximate region shown in $(A, B)$ at higher magnification. Anterior is to the right, posterior to the left. Scale bar, $100 \mu \mathrm{m}$. 
In E10.5 control embryos, CXCL12 is expressed in cranial (Fig. 1B left) and somite regions (inset in Fig. 1B left). In sharp contrast, in mutant embryos CXCL12 expression is markedly decreased but not completely absent in corresponding regions (Fig. 1B right and inset). We have also performed RT-PCR analyses using primers positioned in each of three CXCL 12 exons in $C X C L 12^{+/+}, C X C L 12^{+/}$ ${ }^{\triangle e x 2}$ and $C X C L 12^{\Delta e x 2 / \Delta e x 2}$ samples (Fig. 1C). Our analyses revealed that no transcript is produced from exon 2 in $C X C L 12^{\triangle e \times 2 / \Delta e x 2}$ mutants. However, we have detected some mutant transcript(s) generated from exons 1 and 3 .

\section{CXCL12 $\Delta^{\text {ex2/Lex2 }}$ mutant embryos are characterized by disrupted cerebellar cytoarchitecture}

Since CXCL12 is required for normal cerebellar development (Ma et al., 1998; Zhu et al., 2002), we sought to examine this structure in our CXCL $12^{\Delta e \times 2 / \Delta e \times 2}$ embryos using known EGL molecular markers. To do so, we performed RNA in situ hybridization using a Barh/1 riboprobe and immunofluorescent analyses using Pax6 antibodies. In E16.5 and E18.5 control embryos, the EGL is peripherally localized (Figs. $2 \mathrm{~A}$ and $2 \mathrm{C} / 2 \mathrm{E}$, respectively). In sharp contrast, the mutantEGL appears to extend into the cerebellar parenchyma apparent at both embryonic stages (Figs. 2B, 2D, and 2F).

\section{Initial radial migration of $m D A$ neurons is altered in CXCL12 ${ }^{\operatorname{Lex} 2 / \Delta e \times 2}$ embryos}

CXCL12 global mutant mice exhibit altered initial radial migration of mDA neurons. Accordingly, we examined this phenomenon in our CXCL12 $12^{\Delta e x 2 / \Delta e x 2}$ embryos by using antibodies against the postmitotic mDA neuron marker, tyrosine hydroxylase (TH). In control embryos, upon exiting the ventricular zone around E10.511.5 , young postmitotic mDA neurons emerge in the intermediate zone and transition to the mantle zone where they start expressing TH (Fig. 3A). In contrast, CXCL12 ${ }^{\Delta e x 2 / \Delta e x 2}$ embryos were characterized by some mDA neurons localized within the intermediate zone, indicating that these neurons were setback along their radial migration path (Fig. 3B). As development proceeds, at E13.5 in control embryos, mDA neuron radial migration becomes prominent throughout the rostrocaudal extent of the ventral midbrain midline (Fig. 3 C,E,G, respectively). In contrast, radial migration of mDA neurons in CXCL12 $12^{\Delta e x 2 / \Delta e x 2}$ embryos deviated from the midline and some mDA neurons appeared to be ectopically positioned in the middle region of the mantle zone at all axial levels (Fig. 3D, 3F and $3 \mathrm{H}$ ). This initial obstruction to $\mathrm{mDA}$ neuron radial migration seems to exist transiently since these misplaced migrating neurons become less evident at later embryonic stages (E18.5) as appar-

Fig. 3. Altered radial migration of mDA neurons in $C X C L 12^{\operatorname{sex} 2 / \Delta e \times 2} \mathrm{em}$ bryos. Midbrain coronal sections were labeled for tyrosine hydroxylase (TH). (A,B) Red dotted lines indicate a separation between ventricular (vz; $\mathrm{TH}-$ ), intermediate (iz; $\mathrm{TH}-)$ and mantle $(\mathrm{mz} ; \mathrm{TH}+)$ zones. Arrowhead depicts TH+ neurons ectopically positioned in the mutant iz. (C-H) The $\mathrm{TH}+\mathrm{mz}$ was partitioned into upper (u), middle $(\mathrm{m})$ and lower (I) regions by red dotted lines. Arrowheads indicate increased numbers of TH+ neurons in the middle $(m)$ region of the $m z$ in mutants compared to controls. $(\mathbf{I}, \mathbf{J})$ Substantia Nigra pars compacta (SNpc) and Ventral Tegmental Area (VTA) are apparent in controls (I) while mutant embryos show a few misplaced $\mathrm{TH}+$ neurons $(\mathbf{J})$ (see arrowheads). Insets in $(A, I)$ are DAPI labeled coronal midbrain sections in which the white dotted boxes indicate approximate regions shown at higher magnification in $(A-H)$ and $(\mathrm{I}, \mathrm{J})$, respectively. Scale bar, $100 \mu \mathrm{m}$ ent by similarities in the formation of the Substantia Nigra pars compacta (SNpc) and Ventral Tegmental Area (VTA) in control and mutant embryos (Fig. $3 \mathrm{I}$ and $3 \mathrm{~J}$, respectively).

\section{CXCL12 ${ }^{\Delta e x 2 / \Delta e x 2}$ mutant embryos show derailed migration and disrupted formation of the PGN}

Aberrant PGN migration in global $C X C R 4$ mutants led us to examine formation of this structure in our $C X C L 12^{\Delta \text { ex2/Aex2 }}$ embryos. In control animals at E14.5, newly arrived Pax6+ and Barh/1+PGN neurons begin to settle in the anterior ventral hindbrain. En route to their final destination, PGN neuron migration is also detected in more caudal regions of the hindbrain localized beneath the pial surface. In contrast, numbers of PGN neurons appear to be substantially reduced in the anterior ventral hindbrain of $C X C L 12^{\Delta e x 2}$ $\triangle$ ex2 embryos. Concomitantly, mutant migration appears to be derailed wherein PGN neurons are ectopically located at the midline
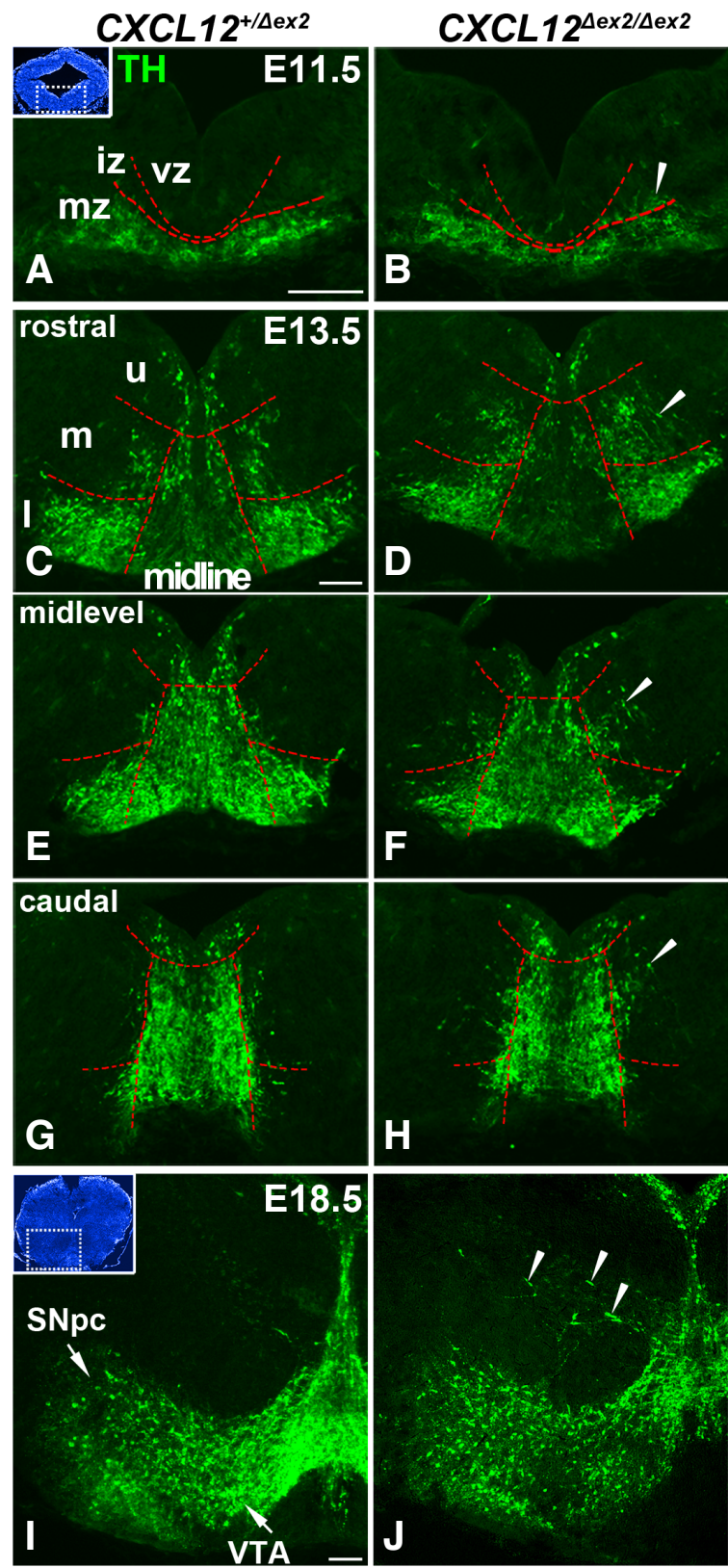
and enter the caudal hindbrain parenchyma (Fig. S4). This early disruption of $P G N$ neuron migration resulted in a drastic reduction of the size of the PGN at E16.5 (Fig. S5).

$P G N, E C N$, and LRN are prominent structures in control embryos at E18.5 (Fig. 4A, 4E and 4G, respectively). Consistent with phenotypes in younger mutant embryos (Figs. S4 and S5), a drastic
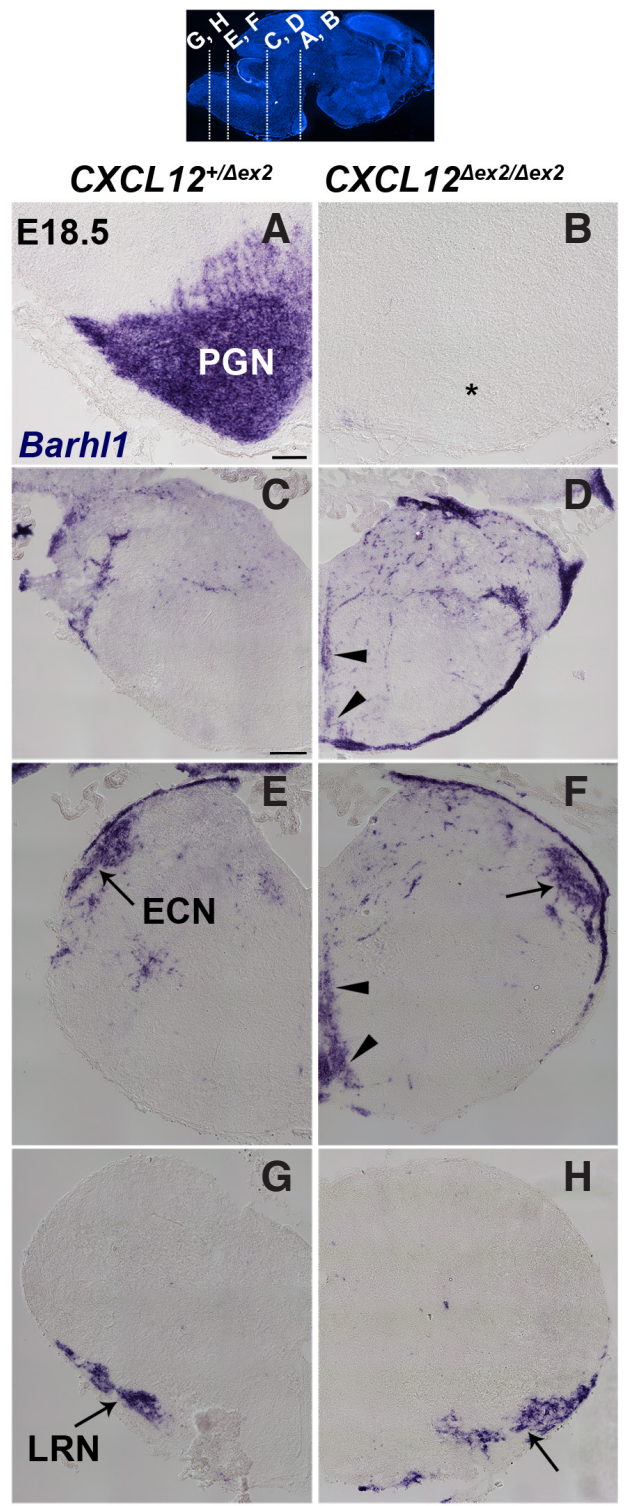

Fig. 4. CXCL12 is required for proper migration of pontine grey nucleus (PGN) neurons. Coronal hindbrain sections were labeled with a Barhl1 riboprobe. Control embryos display prominent PGN anteriorly (A) and external cuneate nucleus (ECN) along with lateral reticular nucleus (LRN) posteriorly (see arrows in (E,G) respectively). Mutant CXCL12 $12^{\Delta \mathrm{ex} 2 / \Delta \mathrm{ex} 2} \mathrm{em}$ bryos exhibit a drastically reduced PGN (see asterisk in (B)) and ectopically localized PGN neurons at the hindbrain midline (see arrowheads in (D,F)). In contrast, the ECN and LRN seem to be unaffected in the mutants (arrows in $(\mathbf{F}, \mathbf{H})$ respectively), when compared to controls $(\mathbf{E}, \mathbf{G})$ respectively. The upper image is a DAPI labeled sagittal section in which white dotted lines indicate approximate positions of coronal sections shown in $(A-H)$. Anterior is to the right, posterior to the left. Scale bars, $100 \mu \mathrm{m}(A, B)$ and $200 \mu \mathrm{m}(\mathrm{C}-\mathrm{H})$. reduction of the PGN is maintained at E18.5 (Fig. 4B). Moreover, migrating mutant neurons appear to ectopically enter the hindbrain parenchyma becoming stalled at the midline (Fig. 4D and 4F, arrowheads). Despite these hindrances to PGN neuron migration, ECN and LRN appear to be intact in CXCL12 ${ }^{\operatorname{\Delta ex2} / \Delta e \times 2}$ embryos (Fig. $4 \mathrm{~F}$ and $4 \mathrm{H}$ ), reminiscent of phenotypes reported in CXCR4 mutants (Vilz et al., 2005; Zhu et al., 2009).

\section{Discussion}

In this report, by using our newly developed CXCL12 floxed line, we have generated $C X C L 12^{\Delta e \times 2 / \Delta e \times 2}$ mutants that were characterized by a marked reduction of CXCL12 transcript. Though our RT-PCR analyses indicated a presence of mutant transcripts, the observed CXCL12 $12^{\Delta \times 2 / \Delta e x 2}$ mutant phenotypes either matched or complemented those that have been previously established for the CXCL12/CXCR4 system. Thus, it is conceivable that although mutant transcripts could be transiently produced, our floxed line is capable of generating a CXCL12 loss-of-function mutation in vivo.

Although previous CXCL12lines have also been generated with loxP sites flanking exon 2 (Ding and Morrison, 2013; Greenbaum et al., 2013; Tzeng et al., 2011), our line was characterized specifically based on neural phenotypes established in the literature. Moreover, we have recapitulated global CXCL12 mutant phenotypes in the developing CNS such as disorganized EGLstructure in the cerebellum and negatively affected radial migration of mDA neurons confirming previously proposed functions of the CXCL12/CXCR4 axis in these systems. Importantly, we reveal that CXCL12 is required for proper migration of PGN neurons in vivo. Furthermore, the striking similarities between PGN phenotypes observed in our CXCL12 ${ }^{\text {sex2/ }}$ ${ }^{\Delta e x 2}$ embryos and global CXCR4 mutants suggest a monogamous relationship between the $\mathrm{CXCL12}$ ligand and its receptor CXCR4 in PGN development.

Taken together, presented results strongly suggest that our newly developed CXCL12 floxed line can be used as a powerful tool to generate a $C X C L 12$ loss-of-function mutation and manipulate CXCL12 signaling in vivo. With the plethora of available cre drivers, conditional mutagenesis of CXCL12could provide for a more detailed understanding of the role of this chemokine in the development of numerous biological systems and processes.

\section{Materials and Methods}

\section{Generation of mice harboring a CXCL12 floxed allele}

A CXCL12 floxed exon 2 mouse line was generated by homologous recombination (inGenious Targeting Laboratory, Inc., Ronkonoma, NY, USA; Supplementary Text and Illustrations). Mice were maintained and sacrificed according to the protocols approved by the Medical College of Wisconsin Institutional Animal Care and Use Committee.

\section{PCR, RT-PCR, RNA in situ hybridization and immunofluorescence}

These analyses were performed as previously described (Joksimovic et al., 2009; Supplementary Text and Illustrations). At least three embryos of each genotype were analyzed for each experiment.

\section{Acknowledgments}

We thank Dr. Richard Miller for providing the CXCL12 riboprobe (Lu et al., 2002). The technical assistance of Sheena L. Faherty, Jenny Grewal, and Donna McAllister is greatly appreciated. This work was supported by startup funding from the Medical College of Wisconsin to M.J. and in part, from philanthropic gifts of the Bobbie Nick Voss Charitable Foundation to M.B.D. 


\section{References}

BAGGIOLINI M (1998). Chemokines and leukocyte traffic. Nature 392: 565-568.

BALABANIAN K, LAGANE B, INFANTINO S, CHOW K Y, HARRIAGUE J, MOEPPS $B$, ARENZANA-SEISDEDOS F, THELEN M, BACHELERIE F (2005). The chemokine SDF-1/CXCL12 binds to and signals through the orphan receptor RDC1 in T lymphocytes. J Biol Chem 280: 35760-35766.

BODEA G, SPILLE J-H, ABE P, ANDERSSON A, ACKER-PALMER A, STUMM R, KUBITSCHECK U, BLAESS S (2014). Reelin and CXCL12 regulate distinct migratory behaviors during the development of the dopaminergic system. Development 141: 661-673.

CRUMPMP, GONG JH, LOETSCHERP, RAJARATHNAM K, AMARAA, ARENZANASEISDEDOS F, VIRELIZIER J L, BAGGIOLINI M, SYKES B D, CLARK-LEWIS (1997). Solution structure and basis for functional activity of stromal cell-derived factor-1; dissociation of CXCR4 activation from binding and inhibition of HIV-1. EMBO J 16: 6996-7007.

DING L, MORRISON S (2013). Haematopoietic stem cells and early lymphoid progenitors occupy distinct bone marrow niches. Nature 495: 231-235.

GREENBAUM A, HSU Y-M S, DAY R, SCHUETTPELZ L, CHRISTOPHER M, BORGERDING J, NAGASAWAT, LINKD (2013). CXCL12 in early mesenchymal progenitors is required for haematopoietic stem-cell maintenance. Nature 495: 227-230.

JOKSIMOVIC M, YUN B A, KITTAPPA R, ANDEREGG A M, CHANG W W, TAKETO M M, MCKAY R D, AWATRAMANI R B (2009). Wnt antagonism of Shh facilitates midbrain floor plate neurogenesis. Nat Neurosci 12: 125-31.

LU M, GROVE E, MILLER R (2002). Abnormal development of the hippocampal dentate gyrus in mice lacking the CXCR4 chemokine receptor. Proc Natl Acad Sci USA 99: 7090-7095.

MA Q, JONES D, BORGHESANI P, SEGAL R, NAGASAWA T, KISHIMOTO T, BRONSON R, SPRINGER T (1998). Impaired B-lymphopoiesis, myelopoiesis, and derailed cerebellar neuron migration in CXCR4- and SDF-1-deficient mice. Proc Natl Acad Sci USA 95: 9448-9453.

NAGASAWAT, HIROTAS, TACHIBANAK, TAKAKURAN, NISHIKAWAS, KITAMURA Y, YOSHIDA N, KIKUTANI H, KISHIMOTO T (1996). Defects of B-cell lymphopoiesis and bone-marrow myelopoiesis in mice lacking the CXC chemokine PBSF/ SDF-1. Nature 382: 635-638.
OBERLIN E, AMARA A, BACHELERIE F, BESSIA C, VIRELIZIER J L, ARENZANASEISDEDOS F, SCHWARTZ O, HEARD J M, CLARK-LEWIS I, LEGLER D F et al., (1996). The CXC chemokine SDF-1 is the ligand for LESTR/fusin and prevents infection by T-cell-line-adapted HIV-1. Nature 382: 833-5.

RODRIGUEZ C, DYMECKI S (2000). Origin of the precerebellar system. Neuron 27: $475-486$

SÁNCHEZ-ALCAÑIZ J, HAEGE S, MUELLER W, PLA R, MACKAY F, SCHULZ S, LÓPEZ-BENDITO G, STUMM R, MARÍN O (2011). Cxcr7 controls neuronal migration by regulating chemokine responsiveness. Neuron 69: 77-90.

TZENG Y-S, LI H, KANG Y-L, CHEN W-C, CHENG W-C, LAI D-M (2011). Loss of $\mathrm{Cxcl12/Sdf-1} \mathrm{in} \mathrm{adult} \mathrm{mice} \mathrm{decreases} \mathrm{the} \mathrm{quiescent} \mathrm{state} \mathrm{of} \mathrm{hematopoietic}$ stem/progenitor cells and alters the pattern of hematopoietic regeneration after myelosuppression. Blood 117: 429-439.

VILZ T, MOEPPS B, ENGELE J, MOLLY S, LITTMAN D, SCHILLING K (2005). The SDF-1/CXCR4 pathway and the development of the cerebellar system. Eur $J$ Neurosci 22: 1831-1839.

WANG Y, LI G, STANCO A, LONG J, CRAWFORD D, POTTER G, PLEASURE S BEHRENS T, RUBENSTEIN J (2011). CXCR4 and CXCR7 have distinct functions in regulating interneuron migration. Neuron 69: 61-76.

YANG S, EDMANL, SÁNCHEZ-ALCAÑIZJ, FRITZN, BONILLAS, HECHT J, UHLÉN P, PLEASURE S, VILLAESCUSAJ, MARÍNO etal., (2013). Cxcl12/Cxcr4 signaling controls the migration and process orientation of A9-A10 dopaminergic neurons. Development 140: 4554-4564.

ZHU Y, MATSUMOTO T, MIKAMI S, NAGASAWA T, MURAKAMI F (2009). SDF1/ CXCR4 signalling regulates two distinct processes of precerebellar neuronal migration and its depletion leads to abnormal pontine nuclei formation. Development 136: 1919-1928.

ZHU Y, YU T, ZHANG X-C, NAGASAWA T, WU J, RAO Y (2002). Role of the chemokine SDF-1 as the meningeal attractant for embryonic cerebellar neurons. Nat Neurosci 5: 719-720.

ZOU Y, KOTTMANN A, KURODAM, TANIUCHI I, LITTMAN D (1998). Function of the chemokine receptor CXCR4 in haematopoiesis and in cerebellar development. Nature 393: 595-599. 


\section{Further Related Reading, published previously in the Int. J. Dev. Biol.}

Expression of $x S D F-1 \alpha, x C X C R 4$, and $x C X C R 7$ during gastrulation in Xenopus laevis Surabhi-Kirti Mishra, Tomoko Nagata, Kazuya Furusawa,Naoki Sasaki and Akimasa Fukui Int. J. Dev. Biol. (2013) 57: 95-100

http://dx.doi.org/10.1387/ijdb.120130af

\section{CXCL14 expression during chick embryonic development}

Christopher T. Gordon, Christine Wade, Inigo Brinas and Peter G. Farlie

Int. J. Dev. Biol. (2011) 55: 335-340

http://dx.doi.org/10.1387/ijdb.103258cg

Developmental expression and regulation of the chemokine CXCL14 in Xenopus Byung-Yong Park, Chang-Soo Hong, Faraz A. Sohail and Jean-Pierre Saint-Jeannet Int. J. Dev. Biol. (2009) 53: 535-540

http://dx.doi.org/10.1387/ijdb.092855bp

Molecular mechanisms controlling brain development: an overview of neuroepithelial secondary organizers

Claudia Vieira, Ana Pombero, Raquel García-Lopez, Lourdes Gimeno, Diego Echevarria and Salvador Martínez

Int. J. Dev. Biol. (2010) 54: 7-20

http://dx.doi.org/10.1387/ijdb.092853cv

The chemokine network in cancer - much more than directing cell movement Hagen Kulbe, Neil R. Levinson, Fran Balkwill and Julia L. Wilson Int. J. Dev. Biol. (2004) 48: 489-496

http://dx.doi.org/10.1387/ijdb.041814hk

5 yr ISI Impact Factor $(2013)=2.879$
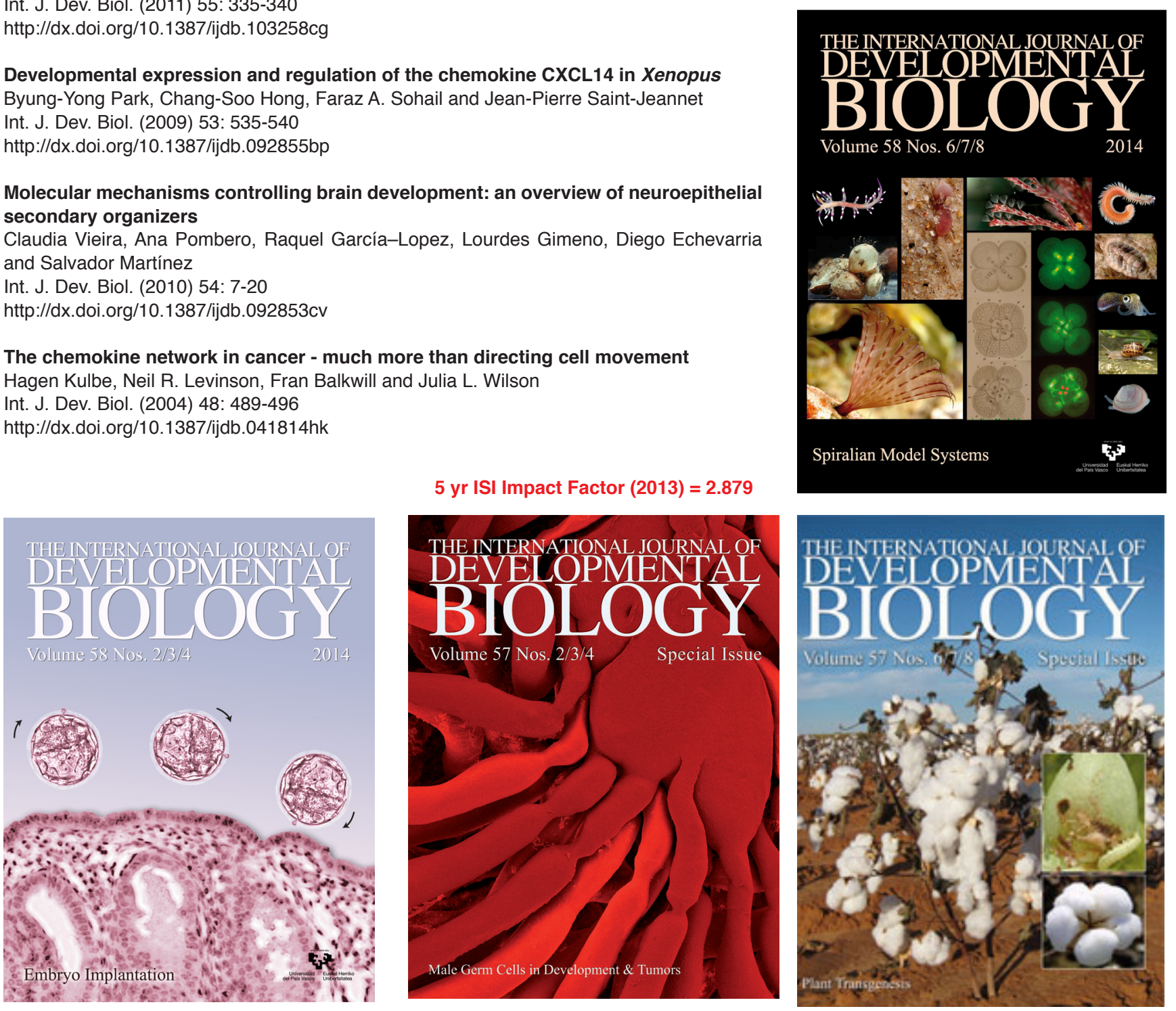